\title{
Urachal-sigmoid fistula associated with diverticular disease
}

\author{
Daniel Rapoport, MD; ${ }^{*}$ Alison Ross, MD; ${ }^{\dagger}$ Vic Goshko, MD; ${ }^{+}$ \\ Iain McAuley, $M D^{\mathcal{E}}$
}

\begin{abstract}
Background: Urachal anomalies rarely present in adulthood. We report the second known case of urachal-sigmoid fistula associated with diverticular disease.
\end{abstract}

Method: We performed a case report and literature review. We searched MEDLINE and PubMed using the search words "urachus," "urachal fistula," "sigmoid colon" and "diverticulosis."

Results: Our literature review revealed 1 previous report of urachal-sigmoid fistula associated with diverticular disease. We reviewed other publications with respect to pathophysiology, diagnosis and management of urachal disease.

Conclusion: Urachal disease that presents in an adult is usually the result of some complication of a urachal anomaly. Various modes of imaging may help confirm the diagnosis, although CT sinography has been recommended and was key in the present case. Management includes eradication of infection and, usually, surgical intervention.

CUAJ 2007; 1(1):52-4

$\mathrm{T}$ he urachus is the embryonic remnant of the communication between the bladder and the umbilicus. ${ }^{1}$ Four categories of urachal disease have been described: urachal sinus, urachal cyst, patent urachus and urachal diverticulum. ${ }^{2,3}$ Urachal anomalies rarely present in adulthood. The remnant may become infected, rupture or fistulize with adjacent viscera. Two cases of urachal-sigmoid fistulae have been described in the literature. One was associated with diverticular disease, while the other was without associated bowel pathology. We report another case of urachal-sigmoid fistula associated with diverticular disease.

\section{Case Report}

LC is a 68-year-old man with type II diabetes and coronary artery disease. He presented with a 5-day history of peri-umbilical pain and erythema. On the fifth day, green discharge and gas passed LC's umbilicus. He experienced no change in bowel habit and had no hematochezia. LC denied any lower urinary tract symptoms. Physical examination revealed peri-umbilical erythema and gaseous umbilical discharge.

The urinalysis was unremarkable. An abdominal CT sinogram revealed an inflamed loop of sigmoid colon adjacent to a urachal remnant. CT sinogram also confirmed a communication between the urachal remnant and the sigmoid colon (Fig. 1, Fig. 2, Fig. 3).

LC was treated with a 6-week course of ciprofloxacin and metronidazole. He subsequently underwent elective resection of the involved segment of the sigmoid colon and excision of the urachal remnant. Gross pathologic examination showed an inflamed portion of sigmoid colon fistulized to the urachus (Fig. 4).

\section{Discussion}

The urachus is an embryonic remnant of the communication between the bladder and the umbilicus. ${ }^{1}$ This communication is usually obliterated between the fourth and fifth month of gestation. It becomes a fibrous cord between the umbilicus and the bladder dome, also known as the median umbilical ligament. ${ }^{2}$ There is a 1 in 5000 incidence of a urachal anomaly on autopsy studies. ${ }^{4}$

Urachal disease is most commonly manifest in the pediatric population, with a variety of clinical presentations. Retrospective reviews at 2 pediatric urologic institutions ${ }^{2,3}$ have described 4 major categories of urachal disease: urachal sinus, urachal cyst, patent urachus and urachal diverticulum. Of these, the urachal cyst has the most potential to present later in life, after being complicated by rupture or infection.

Several options for diagnostic imaging are available to differentiate between different forms of urachal disease. These include abdominal ultrasound, computed tomography, sinography and cystography. The value of each method may vary based on the clinical findings and suspected diagnosis. In patients presenting with umbilical discharge in whom a urachal sinus, patent urachus or 
ruptured urachal cyst is suspected, the sinogram may be most useful. ${ }^{3}$

Although rare, the majority of adult urachal diseases reported in the literature comprise entero-urachal fistulae. Those involving small bowel in patients with Crohn's disease are most commonly reported, while only 2 other cases of urachal-sigmoid fistulae have been reported. ${ }^{5,6}$ One was related to diverticular disease, ${ }^{5}$ while the other was not associated with any bowel pathology. ${ }^{6}$ Both patients were treated surgically with resection of the involved colon segment and excision of the urachal remnant.

Our patient represents the second

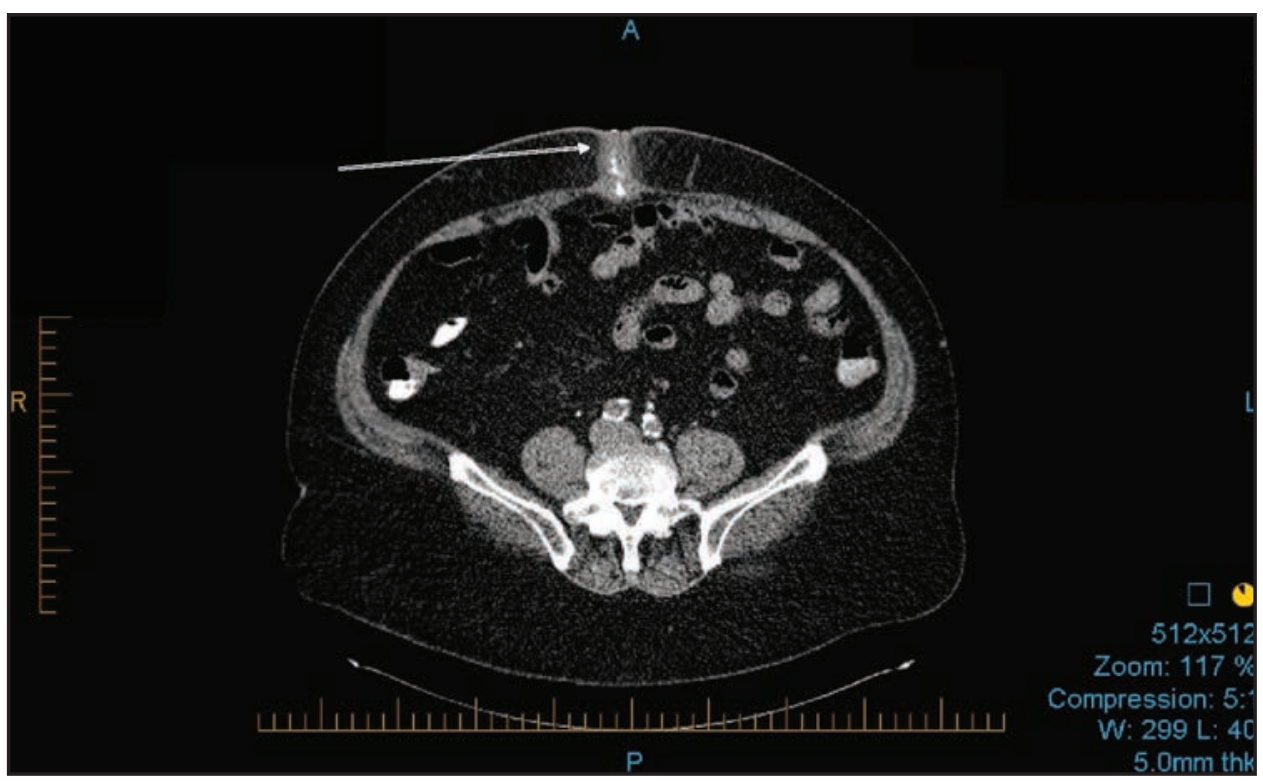

Fig. 1. Axial abdominal CT sinogram.

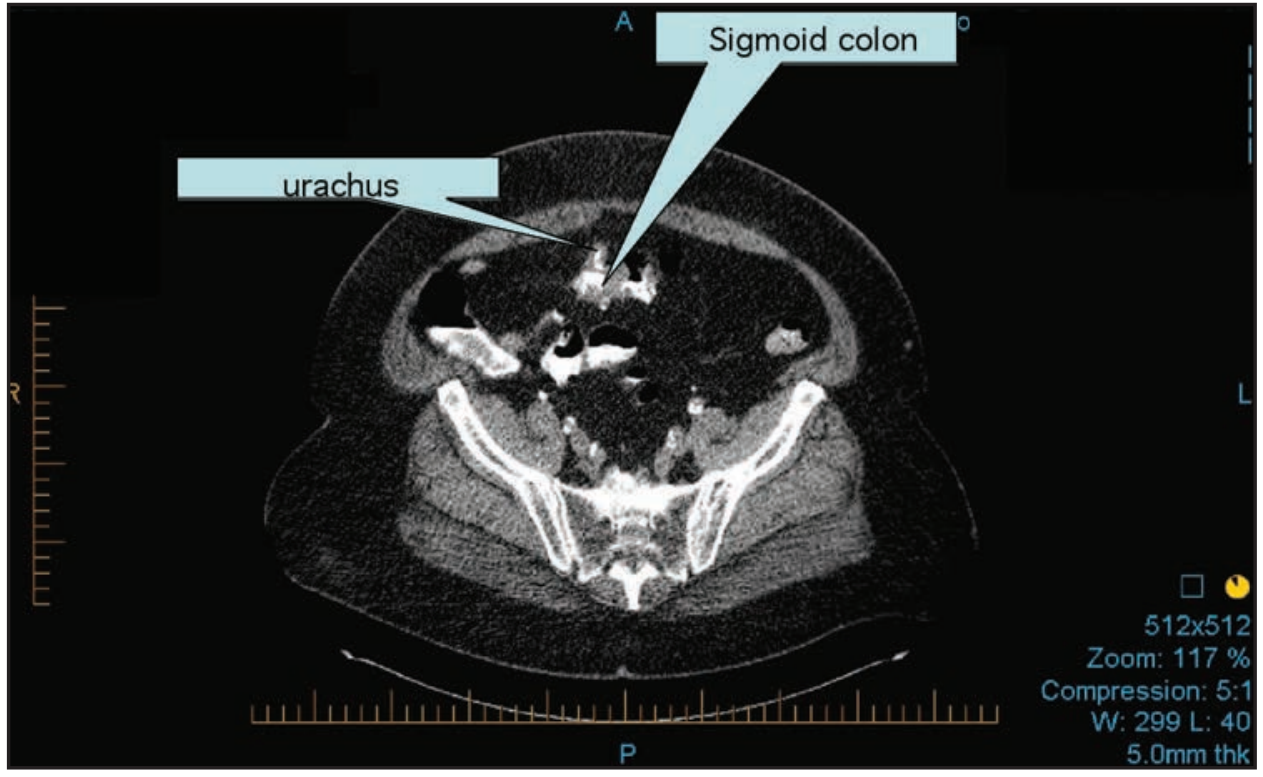

Fig. 2. Axial abdominal CT sinogram demonstrating the communication between the urachal remnant and the sigmoid colon. 


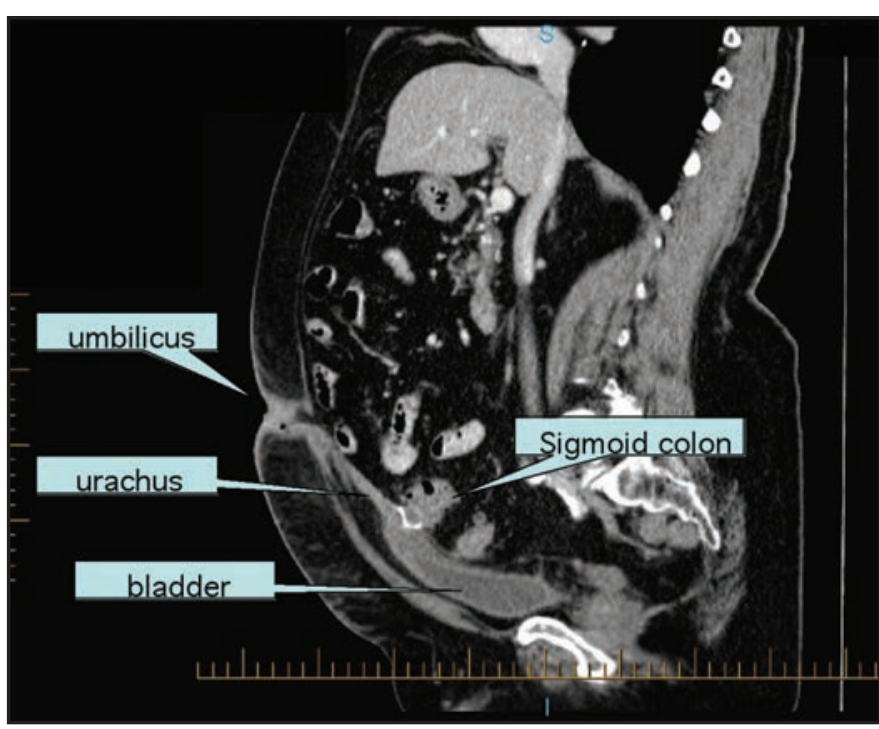

Fig. 3. Sagittal abdominal-pelvis CT sinogram with contrast.

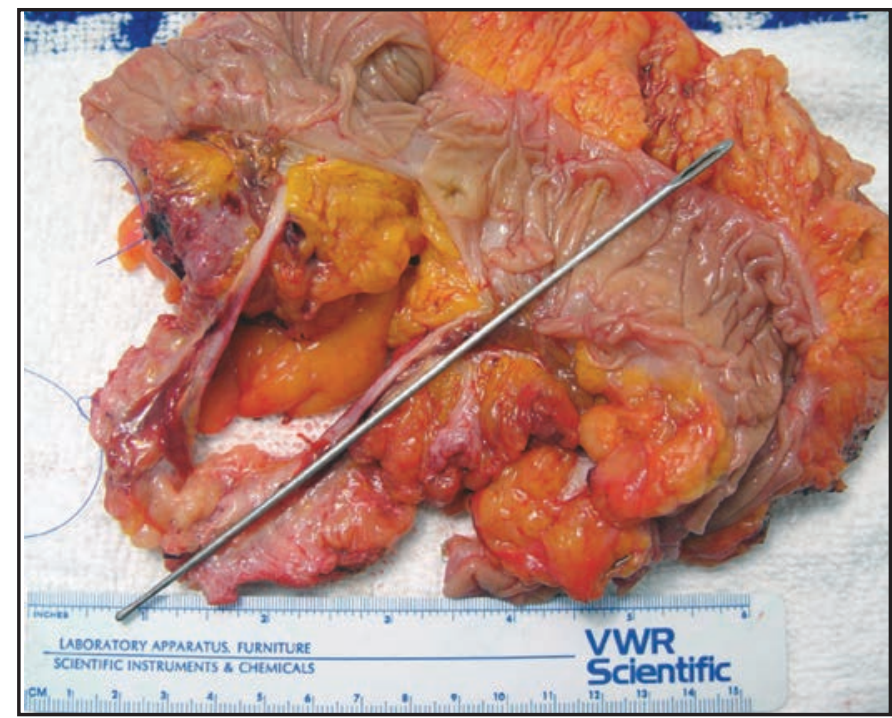

Fig. 4. The inflamed portion of the sigmoid colon fistulized to the urachus. reported case of urachal-sigmoid fistula associated with diverticular disease. The CT sinogram was valuable in establishing the diagnosis in this patient. After eradicating the infection with a 6-week course of antibiotics, we preformed an elective resection of the involved sigmoid colon and urachal remnant, with good results.

From the *Departments of Urologic Sciences and ₹Surgery, University of British Columbia, Vancouver, BC, the 'Division of General Surgery and the $\ddagger$ Department of Emergency Medicine, Victoria General Hospital, Victoria BC, the ${ }^{\ddagger}$ Division of Medical Sciences, University of Victoria, Victoria, BC, and the \pm Department of Emergency Medicine and the ¿Division of Urology, Royal Jubilee Hospital, Vancouver, BC.

This article has been peer reviewed.

Competing interests: None declared.
References

1. Pomeranz A. Anomalies, abnormalities and care of the umbilicus. Pediatr Clin North Am 2004;51:819-27.

2. Mesrobian $\mathrm{HG}$, Zacharias $\mathrm{A}$, Balcom AH, et al. Ten years experience with isolated urachal anomalies in children. J Urol 1997;158:1316-8.

3. Cilento BG, Baver $S B$, Retik AB, et al. Urachal anomalies: defining the best diagnostic modality. Urology 1998;52:120-2.

4. Berman SM, Tolia BM, Laor E, et al. Urachal remnants in adults. Urology 1988; 31:17-21

5. Quek ML, Shpall Al, Daneshmand S. Colourachal fistula in an adult presenting with feculent umbilical drainage. J Urol 2003;170:184.

6. Flanagan DA, Mellinger JD. Urachal-Sigmoid fistula in an adult male. Am Surg 1998;64:762-3.

Correspondence: Dr. lain McAuley, Division of Urology, Vancouver Island Health Authority, Royal Jubilee Hospital, 1952 Bay St., Victoria BC V8R 1J8; iainmcauley@hotmail.com 